\title{
Non-Penetrating Deep Sclerectomy: A Lifetime Dedication and Economical Considerations
}

\begin{abstract}
Ahmed Mostafa Abdelrahman*
Professor of Ophthalmology, Cairo University, Egypt

Egyptian Society for Continuous Ophthalmic Education (EGSCOE), Founder,

Giza specialized eye Center (Glaucoma Management \& Education Center), Owner

*Corresponding Author: Ahmed Mostafa Abdelrahman, Egyptian Society for

Continuous Ophthalmic Education (EGSCOE), Founder, Professor of Ophthalmology,

Cairo University, Egypt. E-mail: Ahmed.mostafa@kasralainy.edu.eg, gizaeyecenter.com
\end{abstract}

Received: March 24, 2021

Published: April 09, 2021

(C) All rights are reserved by Ahmed Mostafa

Abdelrahman.

\section{Abstract}

The present review describes simple economical procedures that were introduced to the literature by the author to facilitate Nonpenetrating Deep Sclerectomy and enhance the efficacy in lowering the intraocular pressure in glaucoma patients.

Keywords: Non-penetrating Deep Sclerectomy (NPDS); Intraocular Pressure (IOP); Schlemm's Canal; Ahmed Suture

\section{Introduction}

Non-penetrating deep sclerectomy (NPDS) is a glaucoma operation characterized by a high safety profile; specifically gradual reduction of the Intraocular pressure (IOP) intraoperatively with avoidance of hazards related sudden decompression of the globe, like hemorrhagic complications of the retina and Choroid. Besides that, the extremely low risk of late intraocular infection is an outstanding advantage. As a result of the high safety profiles, the surgeons are increasingly interested in nonpenetrating glaucoma surgery (NPGS) [1].

NPGS lowers the IOP by allowing aqueous humor to flow through an intact trabeculo-Descemet's membrane (TDM), that is exposed after unroofing of Schlemm's canal (SC) and excision of the deep scleral together with the peripheral cornea in the surgical site. Deep sclerectomy and Viscocanalostomy are the main types of NPGS [2].

NPDS is indicated in open angle glaucoma types; primary open angle glaucoma (POAG), juvenile open angle glaucoma (JOAG), Pe- diatric glaucomas and certain types of secondary glaucomas like Pseudoexfoliation, pigmentary and steroid-induced glaucomas.

Technically, a rectangular $4 * 3 \mathrm{~mm}$, or larger, superficial scleral flap is fashioned using a crescent blade and dissected forward until the corneal periphery is reached. . A smaller deeper second scleral flap is outlined and dissected forward until the Schlemm's Canal (SC) is opened. Identification and opening of SC is the secret of success of NPDS. Beyond this step, the trabecular meshwork (TM) will be identified and percolation of aqueous humor will begin. The end point is creation of a sufficiently percolating TrabeculoDescemet's membrane (TDM) (Figure 1). Peeling of the floor of the SC enhances further percolation, the deep scleral flap is then excised. Because The eye is kept intact, the anterior chamber is not opened and no Peripheral iridectomy, hence the name (NPDS) [2$4]$.

The percolating aqueous will accumulate under the superficial scleral flap forming a lake; the decompression space. Afterwards, the aqueous will flow into the subconjunctival space forming a bleb, through cut ends of SC and occasionally into the supra-ciliary space 


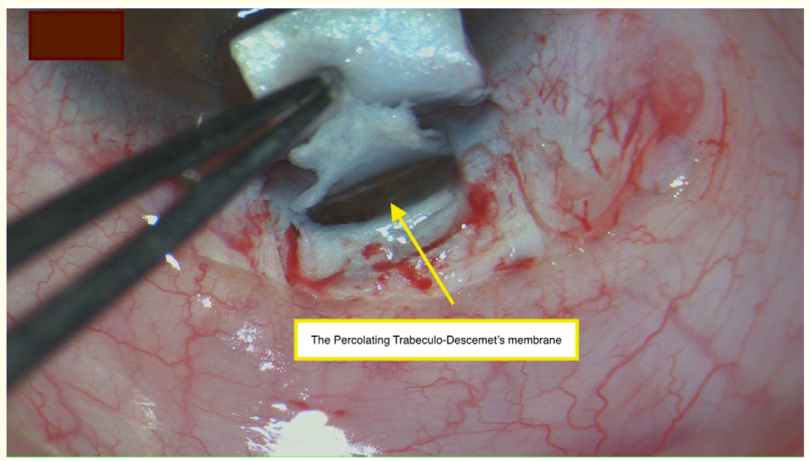

Figure 1: Exposure of the percolating Trabeculo-Descemet's Membrane During NPDS (Abdelrahman AM).

through the thinned out sclera in the decompression space bed. Figure 2 shows NPDS with a superior conjunctival bleb [5].

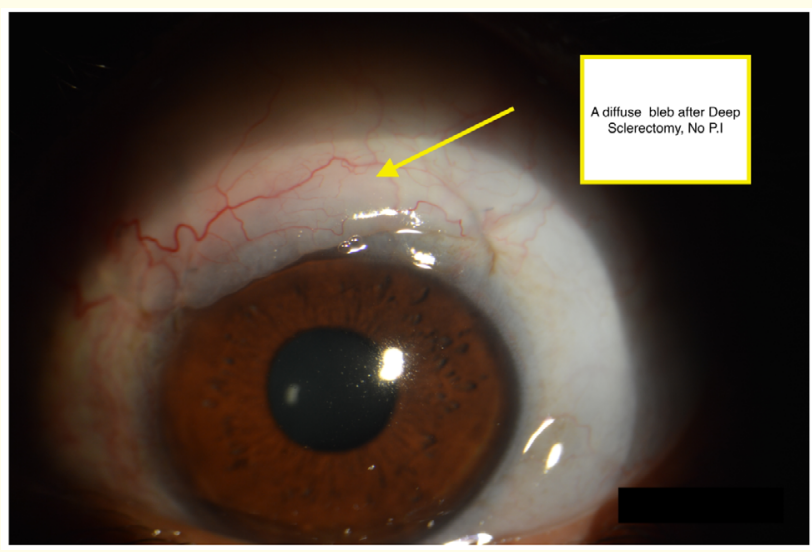

Figure 2: A diffuse conjunctival bleb after NPDS. Notice No peripheral iridectomy (PI) as the eye is not penetrated (Abdelrahman AM).

NPDS has a steep learning curve, this is attributed to the challenges of identifying of SC intraoperatively, dissections above and below SC plane are not desirable. Besides that, the surgeon should be able to manage the IOP postoperatively, should the IOP becomes elevated above the patient's target pressure.
So the big questions and concerns are as follows: 1- how to facilitate SC identification in order to conclude the operation successfully?, 2- how to keep the cut ends of the SC patent postoperatively to enhance the internal drainage through the circumference of the canal? and 3- how to economically keep the decompression space open? anti-metabolites alone might not sufficiently achieve this goal.

\section{Trabeculotome-guided deep sclerectomy}

An essential step in NPGS is the proper dissection of the deep scleral flap to end up in a sufficiently percolating TDM. Deep dissection will leave almost up to $10 \%$ of the sclera over the uvea. In this way, SC will be deroofed with subsequently exposure the anterior trabeculum, Schwalbe's line and peripheral Descemet's membrane. Thus, the outer wall of SC should be included in the excised deep flap. Dietlein., et al. [6] reported that NPDS, even when performed by an experienced glaucoma surgeon, might not show the SC endothelium. They found out that $48 \%$ of the examined deep flaps specimens didn't show SC endothelium, indicating absence of proper unroofing.

In 2005, the Trabeculotome- guided unroofing of SC was introduced as a simple technique that facilitates identification and deroofing SC. A step that could be applied to all NPGS procedures [7].

As the Trabeculotome instrument is routinely used to identify the SC during pediatric glaucoma surgery, I planned to do so during NPDS. A vertical incision is made at the limbus under the superficial scleral flap. The incision is gradually deepened until the SC is cut. The Trabeculotome (Katena) is introduced through this incision into SC and passed horizontally. At the other edge of the planned scleral flap, a direct vertical limbal incision is made over the Trabeculotome to exit the instrument through it. The deep flap is dissected and the SC in incised over the Trabeculotome (Figure 3). In this way, neither superficial nor deep dissections will be done. Surgical details are available [8,9]. This simple economical step This may shorten the learning curve of NPGS.

The Trabeculotome-guided approach was evaluated through a prospective study on 15 eyes with various types of glaucoma, SC endothelium was identified in all the examined specimens. Clinically, the mean IOP was reduced from $26.66 \pm 4.54 \mathrm{~mm} \mathrm{Hg}$ to 12.2 $\pm 3.5 \mathrm{~mm} \mathrm{Hg}$ at the end of a mean follow-up of $9.4 \pm 2.9$ months [9]. 


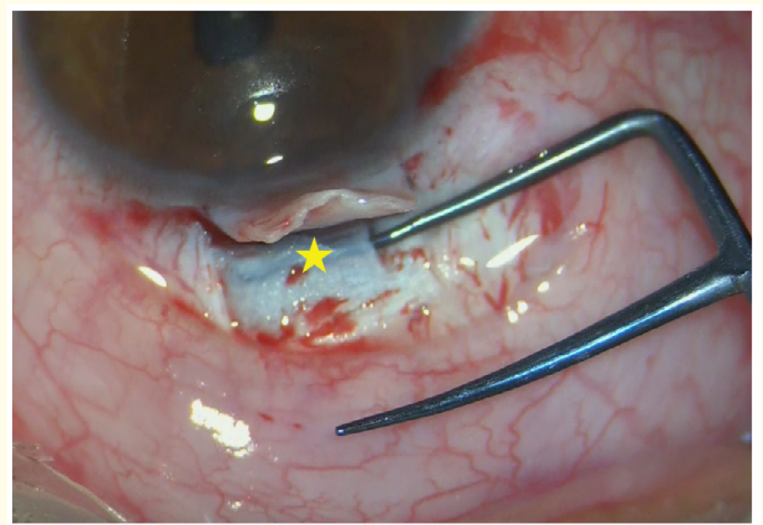

Figure 3: The Trabeculotome is inserted inside Schlemm's Canal during Trabeculotome-guided Deep Sclerectomy (star) (Abdelrahman AM).

Advantages of the described step included the following: 1finding the proper dissection plane for SC unroofing, and avoiding the complications linked to superficial or deep dissections, 2may shorten the learning curve, 3 - avoid too much thinning of the sclera at the surgical site; this may reduce the risk of scleral ectasia $[10,11]$.

\section{Prolene canalostenting during deep sclerectomy}

Another concern is the ability is to maintain the cut ends of SC patent at the surgical site.

We investigated the effect of implanting a 5/0 Prolene suture segment inside SC during NPDS.

A $10 \mathrm{~mm}$ long segment of polypropylene 5/0 (Ethicon, Somerville, NJ, USA) was used as a stent inside SC. (Figure 4 and 5). The full surgical details are described in the literature [12].

We evaluated the IOP drop, postoperative interventions, and complications.

The cases were followed for a mean of $8.1 \pm 4.5$ months. We reported a statistically highly significant drop of the mean IOP and the anti-glaucoma medications. YAG laser goniopuncture was not required in any case. We concluded that, Implantation of a 5/0 Prolene suture in Schlemm's canal during deep sclerectomy was a safe, economical adjunct to NPDS [12].

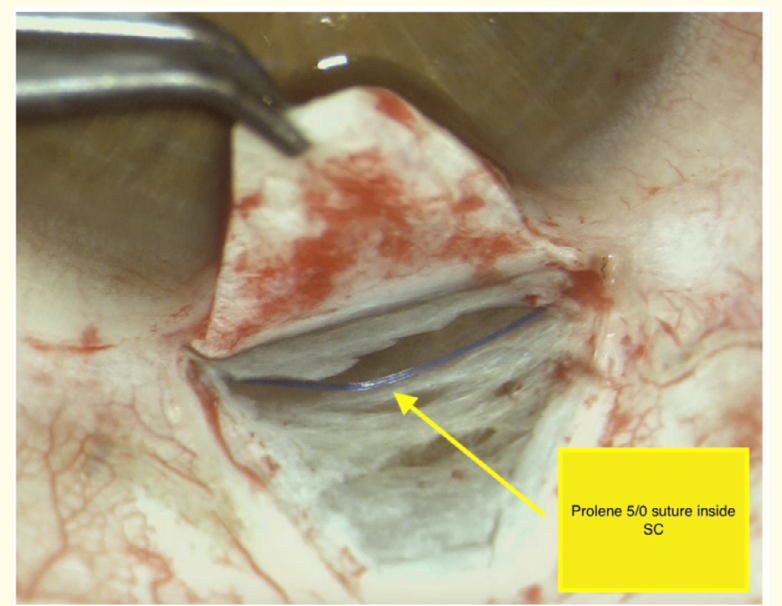

Figure 4: Prolene 5/0 suture inside Schlemm's Canal during Prolene Canalo-stenting (Abdelrahman AM).

Stenting the SC with the 5/0 Prolene segment will keep the SC patent; thereby helping the aqueous flow through the cut ends of SC. Besides that, it may help to maintain the decompression scleral space.

The polypropylene material has a The biocompatible nature that would allow prolonged maintenance of the SC patency in an economical way; $<10$ US\$ per suture $[13,14]$.

Figure 5 shows the blue Prolene suture inside SC during gonioscopic examination. This has the added benefit of localizing the TDM in case YAG goniopuncture is needed for management of postNPDS pressure elevation.

Figure 6 Shows a gonioscopic view of the Prolene suture inside SC seven years after a surgery.

\section{Sutureless deep sclerectomy}

In 2017, another NPDS-modification was investigated where all the sutures were completely avoided. In this way, Sutureless Deep Sclerectomy was introduced to the literature (SDS) [15].

Throughout a prospective study, SDS was evaluated regarding its safety and efficacy. 


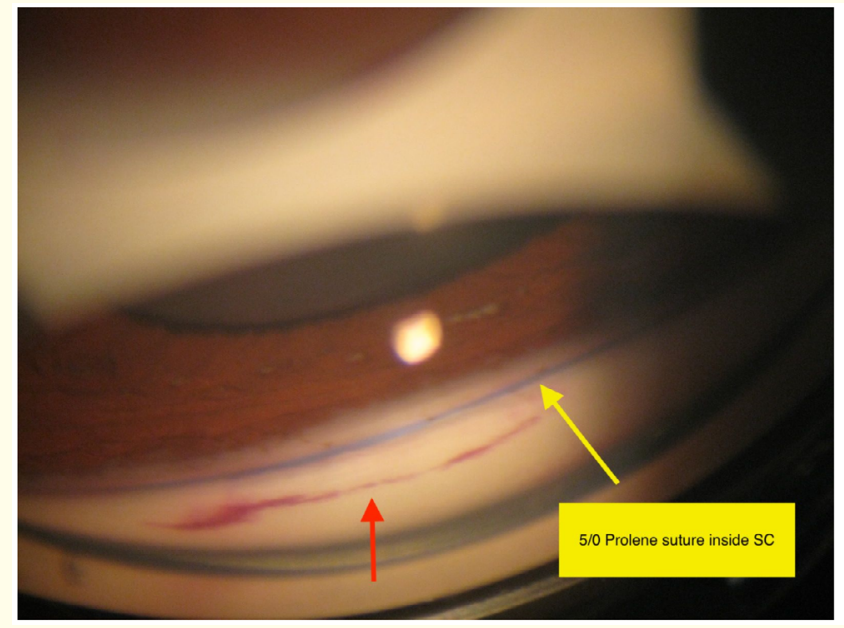

Figure 5: The Prolene suture seen inside SC during gonioscopy (Abdelrahman AM).

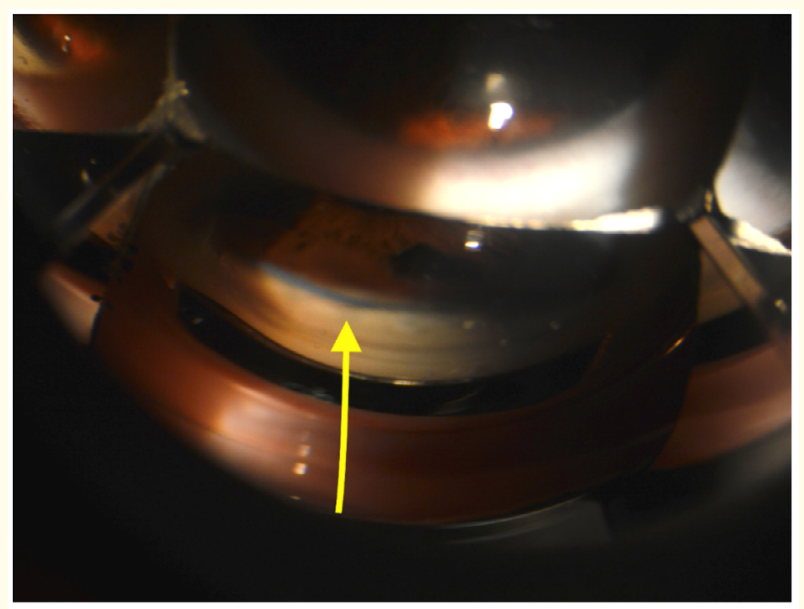

Figure 6: The Prolene suture seen inside SC during gonioscopy 7 years post-operatively (Abdelrahman AM).

We conducted a prospective study on patients medically uncontrolled open-angle glaucomas despite maximally tolerated medical therapy. After excising the deep flap, no sutures were added to the superficial scleral flap nor to the conjunctiva at the conclusion of the surgery. The full technique and results are available in the literature [15].
In summary, After excision of the deep scleral flap and confirming good percolation, the superficial flap was then left without sutures. The conjunctival edges were made adherent using lowpower diathermy and conjunctival incision was tested for any leak using a dry cellulose sponge.

A statistically significant reduction of the intraocular pressure was reported during all the follow-up visits without serious complications. The mean preoperative IOP was $31.72 \pm 10.71 \mathrm{~mm} \mathrm{Hg}$. and $15.07 \pm 3.22 \mathrm{mmHg}$ sixth months postoperatively. The mean IOP was reduced $47 \pm 24.9 \%$ compared with the preoperative IOP. The mean preoperative medication was $2.88 \pm 1.36$ and was reduced to $0.29 \pm 0.469$ at the sixth postoperative month.

We reported few non-serious complications, a temporary dellen in one eye, Tenon cysts in two eyes, minimal conjunctival recession in one eye . No cases of postoperative leak, blebitis or endophthalmitis were reported.

The concept behind SDS is that NPDS a low-flow filtration filtration surgery with mild diffuse conjunctival elevation, so the conjunctival edges remain adherent, without retraction, under the adhesive effect of the low power diathermy.

Other fruitful outcomes of SDS include, shorter operative time, no ocular irritation by the sutures, no need for conjunctival sutures removal which may be difficult in some patients, and obviously more economical.

\section{Sub-flap Mattress Suture During Deep Sclerectomy (Ahmed Suture).}

In 2020, we evaluated a novel simple economical step that enhances the efficacy of deep sclerectomy in lowering the elevated IOP by adding a sub-flap mattress $10 / 0$ Nylon suture after excision of the deep scleral flap. We obtained additional $12.5 \%$ reduction in the IOP compared to the standard surgery [16].

In the literature, various attempts have been made to preserve the scleral space under the superficial scleral flap: collagen implants, reticulated hyaluronic acid implant, nonabsorbable hydrophilic acrylic implant, and viscoelastic implant [17]. Many of those modifications are costly and not available in our practice. Other NPDS- enhancing procedures include, antimetabolites [18], Canaloplasty with insertion of a circumferential tightening Prolene 
10/0 was also described [19,20]. Other reports described adding trabeculectomy [21,22], trabeculotomy [23].

The surgical details of are available in the literature [16]. In summary, A 10/0 nylon suture (Ethilon) ${ }^{\circledR}$ mattress suture that extended $2 \mathrm{~mm}$ beyond the superficial scleral flap edge was secured under the flap (Figure 7 and 8). The suture is non-absorbable and widely available [24].

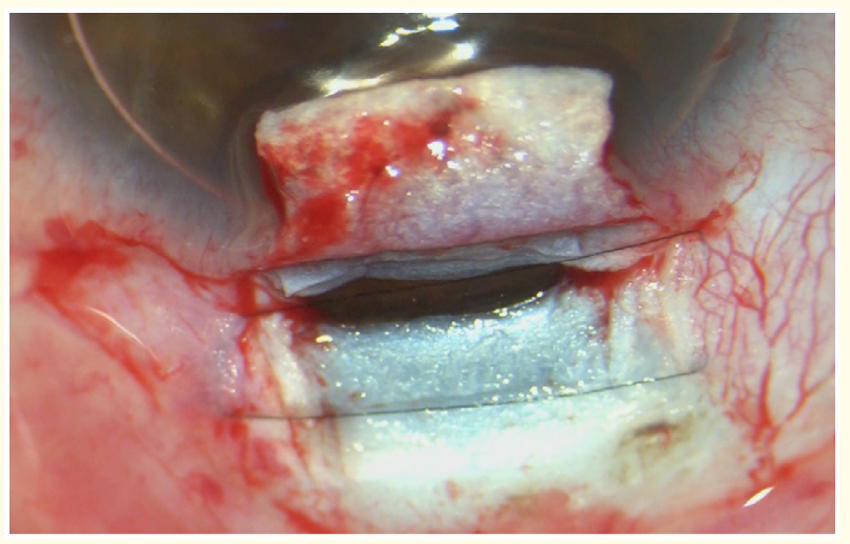

Figure 7: Sub-flap 10/0 nylon mattress suture (Abdelrahman AM).

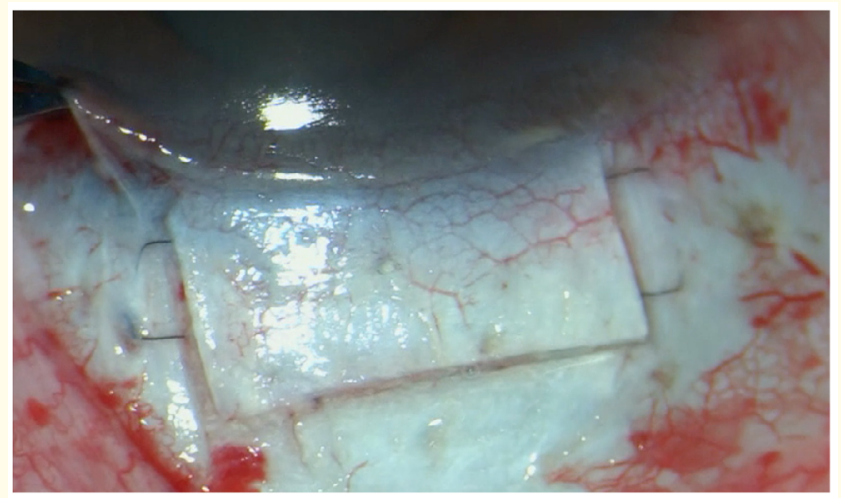

Figure 8: The superficial scleral flap is lifted by the sub-flap mattress $10 / 0$ Nylon suture that extends beyond the edges of the flap (Abdelrahman AM).
Through a prospective clinical trial, we enrolled 29 eyes from 20 patients with open angle glaucoma not controlled on maximum medical treatment. They were randomized into two groups: 14 eyes underwent modified deep sclerectomy (by adding sub-flap mattress suture) and 15 eyes underwent standard deep sclerectomy. Both groups were followed up for 3 months and results were compared.

The intra-ocular pressure was significantly lower in the subflap mattress group and the percentage drop of the IOP from the pre-operative pressure after 3 months was significantly larger in the sub-flap mattress group $(52.9 \% \pm 13.1)$ than in the standard technique group $(40.5 \% \pm 10.3)$.

The enhanced IOP-lowering effect of this single mattress suture (Ahmed Suture) is explained by the following mechanisms: 1- mechanical elevation of the proximal part of the superficial scleral flap, which in turn enhance the aqueous percolation , 2- slight elevation of the scleral flap, without sutures at the edges, would induce some tissue separation, thereby enhancing aqueous movement to the subconjunctival space, 3- tightening the suture by the tangential traction to the limbus would widen the TDM. 4- the proximal limb lies of the suture lies in the Schlemm's canal, this helps visualization of the TDM should YAG goniopuncture is needed.

\section{Conclusion}

In conclusion, over more than 15 years of continuous efforts, I was fortunate to introduce to the literature few steps during deep sclerectomy. Those innovations helped me in better understanding of the indications, surgical anatomy, technique and outcomes of the non-penetrating deep sclerectomy. In addition, the IOP-lowering effect was enhanced through economical materials that were handy.

A YouTube link (Author's video ):

https://youtu.be/u-ijPTrl5-k

\section{Bibliography}

1. Mermoud A., et al. "Comparison of deep sclerectomy with collagen implant and trabeculectomy in open-angle glaucoma". Journal of Cataract and Refractive Surgery 25 (1999): 323-331.

2. Mermoud A. "Evolution of non-penetrating glaucoma surgery". In: Mermoud A, Shaarawy T, Rich R, eds, Non-Penetrating Glaucoma Surgery. London, Martin Dunitz (2001): 13-20 
3. Carassa RG., et al. "Viscocanalostomy versus trabeculectomy in white adults affected by open-angle glaucoma; a 2-year randomized, controlled trial". Ophthalmology 110 (2003): 882887.

4. Stegmann RC. "Visco-canalostomy: a new surgical technique for open angle glaucoma”. An Inst Barraquer 25 (1995): 229-232.

5. Johnson DH and Johnson M. "How does non-penetrating glaucoma surgery work?" In: Mermoud A, Shaarawy T, Rich R, eds, Non-Penetrating Glaucoma Surgery. London, Martin Dunitz (2001): 33-55.

6. Dietlein TS., et al. "Variability of dissection depth in deep sclerectomy: morphological analysis of the deep scleral flap". Graefe's Archive for Clinical and Experimental Ophthalmology 238 (2000): 405-409.

7. Abdelrahman AM. "Trabeculotome-guided unroofing of Schlemm's Canal". Journal of Cataract and Refractive Surgery 31.1 (2005): 238-240.

8. Freedman SF. "Medical and surgical treatments for childhood glaucomas". In: Epstein DL, Allingham RR, Schuman JS, eds, Chandler and Grant's Glaucoma, 4th ed. Baltimore, MD, Williams and Wilkins, (1997): 609-622.

9. Abdelrahman AM. "Trabeculotome-guided Deep Sclerectomy. A pilot Study". American Journal of Ophthalmology 140.1 (2005): 152-154.

10. Mermoud A and Ravinet E. "Surgical technique". In: Mermoud A, Shaarawy T, Rich R, editors, Non-penetrating glaucoma surgery. London, United Kingdom: Martin Dunitz (2001): 97-108.

11. Milazzo S., et al. "Scleral ectasia as a complication of deep sclerectomy". Journal of Cataract and Refractive Surgery 26 (2000): 785-787.

12. Abdelrahman AM and El-Sayed YM. "Prolene Canalostenting During Deep Sclerectomy: a pilot Study". Middle East African Journal of Ophthalmology 22.4 (2015): 514-516.

13. Ates H., et al. "Deep sclerectomy with a nonabsorbable implant (T-Flux): Preliminary results". Canadian Journal of Ophthalmology 38 (2003): 482-488.
14. Grieshaber MC., et al. "Comparing two tensioning suture sizes for 360 degrees viscocanalostomy (canaloplasty): A randomised controlled trial". Eye (Lond) 24 (2010): 1220-1226.

15. Abdelrahman AM., et al. "Sutureless Deep Sclerectomy. A preliminary Report". Journal of Glaucoma 26.11 (2017): e255e256.

16. Abdelrahman AM and Habib MM. "Sub-flap mattress suture with Deep Sclerectomy: A novel step". Journal of Glaucoma 29.11 (2020): e127-e129.

17. Tuesta SD and Stribu O. "Implants in Deep Sclerectomy". In: Shaarawy T, Dada T, Bhartiya S, eds. ISGS Textbook of Glaucoma Surgery. Jaypee Brothers Medical Publishers (P) Ltd. New Delhi, India (2014): 190-197.

18. Al Obeidan SA. "Incidence, efficacy and safety of YAG laser goniopuncture following nonpenetrating deep sclerectomy at a university hospital in Riyadh, Saudi Arabia". Saudi Journal of Ophthalmology 29.2 (2015): 95-102.

19. Riva I., et al. "Canaloplasty in the treatment of open-angle glaucoma: a review of patient selection and outcomes". Advances in Therapy 36.1 (2019): 31-43.

20. Grieshabor M. “Canaloplasty with Suture Stenting”. In: In: Shaarawy T, Dada T, Bhartiya S, eds. ISGS Textbook of Glaucoma Surgery. Jaypee Brothers Medical Publishers (P) Ltd. New Delhi, India (2014): 203-208.

21. Kayikcioglu OR., et al. "Trabeculectomy combined with deep sclerectomy and scleral flap suture tension adjustment under an anterior chamber maintainer: a new modification of trabeculectomy". International Ophthalmology 30.3 (2010): 271-277.

22. Feusier M., et al. "Deep sclerectomy combined with trabeculectomy in pediatric glaucoma". Ophthalmology 116.1 (2009): 30-38.

23. Rho SH., et al. "Surgical Outcomes of Combined Deep Sclerectomy With Trabeculotomy and Trabeculectomy With Trabeculotomy in Pediatric Glaucoma". Investigative Ophthalmology and Visual Science 51.13 (2010): 623. 
24. Rupp J. “Ophthalmic Suturing 101”. AAO. (2018).

\section{Assets from publication with us}

- Prompt Acknowledgement after receiving the article

- Thorough Double blinded peer review

- Rapid Publication

- Issue of Publication Certificate

- High visibility of your Published work

Website: www.actascientific.com/

Submit Article: www.actascientific.com/submission.php

Email us: editor@actascientific.com

Contact us: +919182824667 